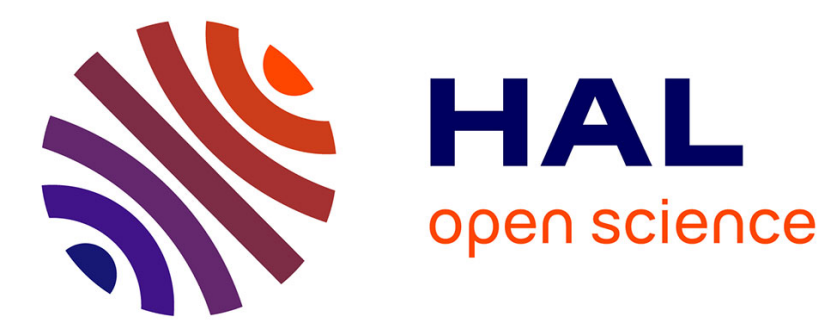

\title{
Le phénomène magnéto-calorique
}

Pierre Weiss

\section{- To cite this version:}

Pierre Weiss. Le phénomène magnéto-calorique. J. Phys. Radium, 1921, 2 (6), pp.161-182. 10.1051/jphysrad:0192100206016100 . jpa-00204281

\section{HAL Id: jpa-00204281 https://hal.science/jpa-00204281}

Submitted on 1 Jan 1921

HAL is a multi-disciplinary open access archive for the deposit and dissemination of scientific research documents, whether they are published or not. The documents may come from teaching and research institutions in France or abroad, or from public or private research centers.
L'archive ouverte pluridisciplinaire HAL, est destinée au dépôt et à la diffusion de documents scientifiques de niveau recherche, publiés ou non, émanant des établissements d'enseignement et de recherche français ou étrangers, des laboratoires publics ou privés. 


\section{LE JOURNAL DE PHYSIQUE \\ $\mathbf{E} \mathbf{T}$}

\section{LE RADIUM}

\section{LE PHÉNOMENE MAGNÉto-Galorique ( $1 /$,}

Par M. Pierre WeISS professeur à l'Université de Strasbourg.

Le phénomène magnéto-calorique a été rencontré à l'occasion d'un travail dont les premiers débuts remontent à 1907. G'est un ensemble de recherches expérimentales que j’ai déjà exécutées plusieurs fois sans que, malgré la précision accrue, elles m'aient donné satisfaction. En 1917, M. Piccard, mon collaborateur de bien des années, et moi-mème, avons remis une fois de plus ces recherches sur le chantier. Ce travail n'est pas encore achevé et ce que je vais vous présenter, ce n'est pas le travail luimème, c'est un sous-produit, une découverte accidentelle faite au cours des derniers efforts tentés pour le mener à bonne fin.

En disant que nous avons travaillé pendant si longtemps dans un ordre d'idées voisin de celui qui va nous occuper, je suis amené à faire un aveu. La découverte dont je vais vous parler, on pouvait la faire en écrivant une seule ligne de calculs. On en avait, en effet, tous les éléments sous la main et ceux -qui, comme mes collaborateurs et moi-mème, s'occupaient de ces questions et avaient l'habitude de manier ces éléments, ont donc été fautifs de ne pas apercevoir cette chose si simple. Cette ligne de calculs, nous l'écrirons tout à l'heure. Auparavant, si vous le voulez bien, nous commencerons par suivre le chemin plus long que j'ai suivi moi-mème.

Mais d'abord, quel est ce travail qui a été effectué six fois avec une précision toujours croissante, toujours insuffisante et que nous n'avons pas encore publié? C'est la topographie exacte d'une fonction de deux variables, de. l'état d'aimantation du nickel dans sa dépendance du champ et de la température. Et, si nous avons recherché une si haute précision, c'est parce qu'il y a des questions de principe à résoudre à l'occasion el par le moyen de cette topographie. Je voudrais d'abord vous présenter les idées directrices de ce travail.

Ces questions de principe se rattachent à l'hypothèse du champ molé-

(1) Conférence faite le 28 février 1920, aux Instituts Solvay à Bruxelles.

Le JOURNAl de Physique et Le radium. - SÉrie vi. - tone II. - JUin 1921. - N 6.11 
culaire. Vous vous rappelez qu'avant que cette hypothèse eùt été formulée, Langevin avait établi une théoric du magnétisme faible, du paramagnétisme, qui donne la loi de l’aimantation cles gaz paramagnétiques, et qui s'applique aussi aux solutions des sels paramagnétiques.

Si nous appelons :

$\sigma_{o}$, la saturation absolue. c'est-à-dire le moment magnétique par unité de masse quand tous les aimants moléculaires sont parallèles;

$\sigma$, l'aimantation, c'est-à-dire le moment magnétique par unité de masse correspondant à la valeur a de la variable; la signification de cette variable étant donnée par

$$
a=\frac{\sigma_{0} H}{R T}
$$

où $H$ est le champ magnétique exercé sur la substance,

$R$, la constante des gaz parfaits rapportée à l'unité de masse $\left(^{1}\right)$;

la loi de Langevin s écrit :

$$
\frac{\sigma}{\sigma_{0}}=\operatorname{coth} a-\frac{1}{a}
$$

Cette loi est représentée par la courbe fig. 1. Elle rend compte des propriétés des corps paramagnétiques. En la discutant, on s'est apercu

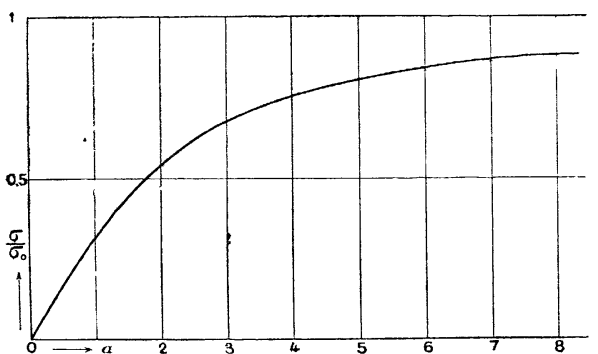

Fig. 1. qu’en général tout ce que l'on peut faire avec les champs actuellement réalisables, c'est d'en parcourir une petite partie dans le voisinage de l'origine, pratiquement la tangente à l'origine, et que la saturation n'est pas accessible. Il faut déjà, comme l'a fait récemment Kamerlingh Onnes dans l'étude magnétique du sulfate de gadolinium $\left({ }^{2}\right)$, descendre très près du zéro absolu pour observer, avec les champs dont on dispose, une courbure sensible et recueillir des indices du voisinage de la saturation.

Cette théorie, je l'ai étendue aux ferromagnétiques par l'hypothèse ‘du champ moléculaire, qui, sous sa première forme, s'exprime de la manière suivante. Considérons l'ensemble des molécules d'une substance aimantée. A l'intérieur de cette substance, il règne un certain champ magnétique qui p. 27 .

(1) Donc $R M=83,15 \times 10^{6}$, où $M$ est la masse moléculaire.

(2) Kinerlingh Onnes, Proc. Acad. Amsterdam, 1914, p. 16 I et Comm. Leiden, n. 140, 
tend à orienter chaque molécule en agissant sur le moment magnétique dont elle est le porteur. Ce champ est proportionnel à l'aimantation $\sigma$ et dirigé comme elle. Il a pour expression

$$
H_{m}=n \sigma ;
$$

où $n$ est une constante et $\sigma$, l'aimantation ou moment magnétique de l'unité de masse. On peut dire que le champ moléculaire est l'action que l'ensemble des molécules aimantées exerce sur l'une d'entre elles (1).

Pour étudier une substance on fait, en général, agir sur elle un certain champ extérieur $\boldsymbol{H}_{\boldsymbol{e}}$. Le champ total $\boldsymbol{H}$ est égal à la somme des deux champs extérieur et moléculaire

$$
H=H_{e}+H_{m}
$$

Voilà les données du problème. Je vais ètre très bref quant-à la manière dont les conséquences s’en déduisent. Considérons d'abord le cas du champ extérieur nul. On peut représenter graphiquement les conditions imposées à la substance. Prenons une température déterminée; nous traçons, pour cette température, la courbe de Langevin qui devient une relation entre $\sigma$ et le champ $\boldsymbol{H}$.

En l'absence d'un champ extérieur, le champ moléculaire existe seul. Représentons aussi la relation entre ce champ et l'intensité d'aimantation. Puisque la quantité $n$, dans la formule (3). est constante, nous avons une droite passant par l'origine.

L'aimantation, qui devra satisfaire aux deux conditions, sera donnée par le point d'intersection A et une discussion plus attentive montre que le point que nous trourons ainsi représente un état stable dans lequel la matière se met spontanément. Par contre, le point d’intersection à l'origine représente l'état non magnétique instable. C'est le moment magnétique

(1) S'il est commode et intuitif de se représenter le champ moléculaire comme un véritable champ magnétique, cette hypothèse est inutilement spécialisée et il y a même des difficultés très sérieuses à admettre que les actions mutuelles d'orientation des molécules douées de moment magnétique soient réellement de nature magnétique. La nature du champ moléculaire serait sans doute intéressante à connaître mais, pour l'objet que nous poursuivons ici, il suffit d'en posséder une expression analytique. On peut conserver, et nous conserverons celle du texte ci-dessus qui fait image. Mais elle pourrait aussi être remplacée par :

$$
H_{m}=-\frac{\partial U}{\partial \sigma}
$$

où $U$ est l'énergie de la substance. Cette dernière définition comprend comme cas particulier la définition (3). Elle a l'avantage d'être indépendante de toute hỳpothèse sur la nature des forces. 
par unité de masse dans l'état stable que j'ai appelé aimantation spontanée.

Cette notion impose une conception nouvelle de ce qu'est un morceau

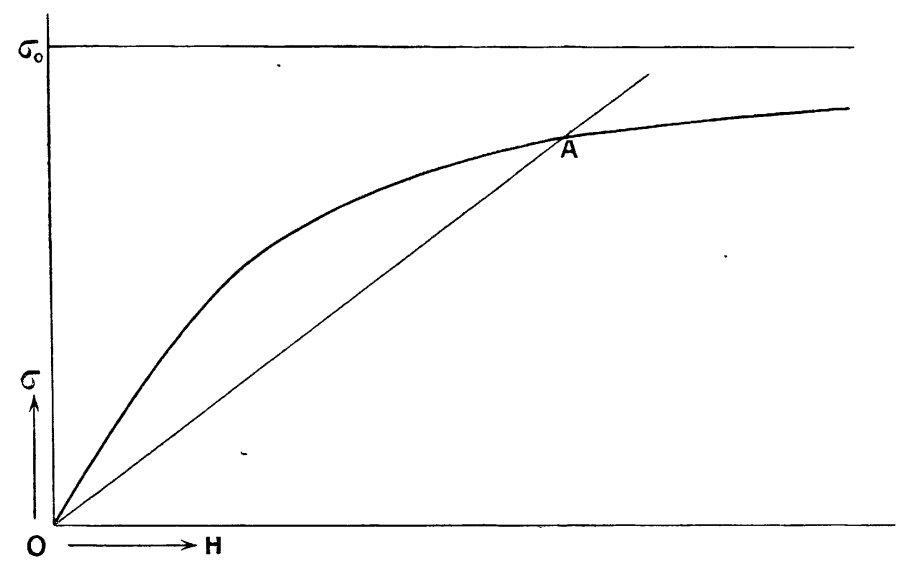

Fig. 2. de fer nonaimanté. Liidée que l’on s'en fait habituellement est, qu'il est quelque chose de différent du fer aimanté, que l'aimantation se produit effectivementau moment où le champ extérieur entre en jeu.

Mais, si l'état aimanté est seul stable, il doit se produire de lui-mème sans intervention, el c'est l'apparence non aimantée qu'il faut expliquer. A cet effet, il faut faire appel à un autre ordre didées, à des considérations de statistique.

La direction dans laquelle. spontanément, l'aimantation se produit, n'est indiquée par rien dans notre théorie. Elle sera donc déterminée par des conditions accessoires qui nont pas été mentionnées et qui peuvent ètre les menus accidents de la substance, les petites discontinuités, les petites cavités qui s'y trouvent et surtout, sans doute, les propriétés anisotropes des cristaux dont elle est composée. Il y a donc. dans un morceau de fer, des régions dans lesquelles l'aimantation est dirigée dans un sens, d'autres où elle est dirigée en sens contraire, d'autres encore où elle a une direction qui n'a aucun rapport avec les premières. En résumé, l'effet d'ensemble est une substance qui semble n'ètre pas aimantée du tout. L'aimantation, telle que les ingénieurs la pratiquent, consiste alors non pas à modifier d'une manière appréciable l'état d'aimantation spontanée, mais à surmonter ces petites causes accessoires que nous n'avions pas jugées dignes d'entrer dans la théorie et à rapprocher ainsi du parallélisme l'aimantation des différentes parties de la substance.

L'aimantation observable, qui est la résultante géométrique des aimantations partielles, ne peut dépasser celle qui correspond au parallélisme parfait. Cette limite est la saturation à la température considérée. Elle ne doit pas ètre confondue avec la saturation absolue ou Langevinienne $\sigma_{o}$ 
dont elle diflère de tout l'effet de l'agitation thermique. En réalité, ce n'est que par approximation rque l'on peut parler de cette limite de l'aimantation à une température déterminée. Des champs suffisamment grands, en général inaccessibles, permettraient de s'approcher à toute température de la saturation absolue $\sigma_{o}$. Avec cette approximation, lorsqu à une certaine température l'expérience donnera nettement une aimantation à saturation celle-ci sera aussi l'aimantation spontanée à cette température. Et, en fait, celte saturation se détermine sans ambiguité tant que la température n'est pas trop voisine du point de Curie que nous allons définir.

Faisons maintenant, à une autre température, la mème détermination graphique de l'aimantation spontanée. Nous voyons que, dans l'une des équations, la température figure et que, dans l'autre, elle ne figure pas. Si nous prenons une température plus élevée, nous aurons à tracer la loi de Langevin à une échelle agrandie des champs, par suite du fait que la température est au dénominateur de la variable $a$, et nous trouverons par l'intersection avec la droite (fig. 2) une aimantation spontanée plus petite. A une certaine température $\Theta$, le point d'intersection ayant rejoint l'origine, l'aimantation spontanée sera nulle.

En utilisant les résultats de cette construction graphique, nous pourons tracer la loi de variation de l'aimantation spontanée en fonction de la température, et cette loi est aussi celle de la variation de l'aimantation à saturation. Ge premier résultat de la théorie du champ moléculaire est représenté dans la fig. 3. Ont été portés en abscisses, les rapports des températures absolues à la température $\Theta$ où l'aimantation spontanée s'annule et en ordonnées, les rapports $\sigma / \sigma_{0}$.

On donnait autrefois à cette température $\Theta$ le nom impropre de point de trans-

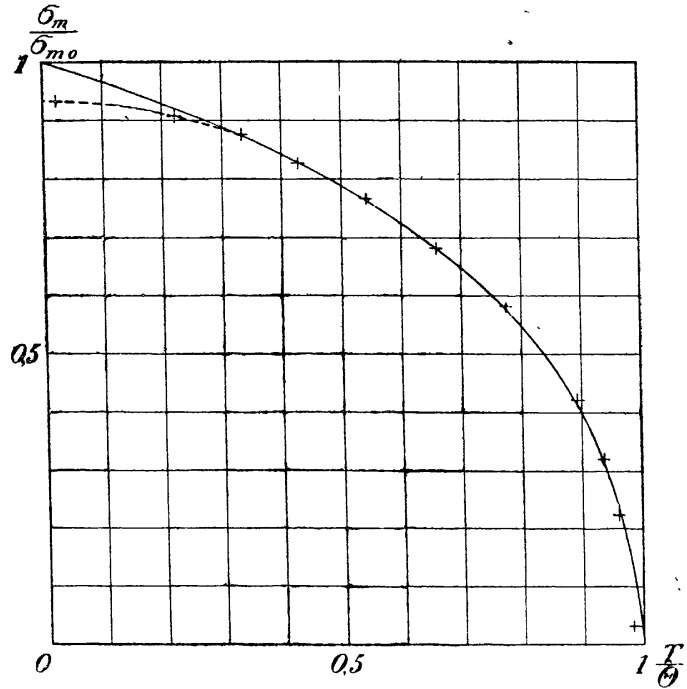

Fig. 3. formation. Maintenant, on l'appelle point de Curie, pour rappeler le travail de Pierre Curie, qui a fait époque dans l'histoire du magnétisme.

Dans la fig. 3, la courbe représente la loi théorique et les points mar- 
qués le résultat de mesures faites sur la magnétite. L’accord est remarquable dans le grand intervalle entre la tempéralure ordinaire et le point de Curie. Aux basses lempératures seulement il y a un écart sensible, dù sans doute à un changement d'état, de nature inconnue. G'est donc un réel succès pour la théorie.

Cette théorie a remporté d'autres succès. Elle a permis de reconnaìtre ce qui se passe au dessus du point de Curie. Si nous représentons, dans cette région, l'inverse du coefficient d'aimantation, $1 / \%$, en fonction de la lempérature, la théorie donne une ligne droite passant par le point de Curie. Et, en effet, on trouve dans l'étude des substances ferromagnéliques au dessus du point de Curie, d'excellentes vérifications de cette loi linéaire.

Enfin une vérification importante a ćté acquise par la décourerte de la nature exacte de lanomalie thermique des corps ferromagnétiques. On savait depuis longtemps qu'il y a quelque chose de spécial dans la loi suivant laquelle il faut fournir de la chaleur aux ferromagnétiques pour produire une élévation de lempérature. On parlait couramment, sans préciser davanlage, de chaleur de transformation.

On ne s'était pas aperçu, en prenant les choses en gros, qu'il ne s'agit pas d'une quantité de chaleur absorbée à une température déterminée, comme c'est le cas pour les changements d'état. mais d'une absorption de chaleur qui s'étend à un intervalle de tempéralures considérable. Et en elfet, un calculfon dé surle champ moléculaire montrequela chaleur spécifiquedoit èlre augmentée d'un terme additionnel dans toute la région ferromagnétique.

Représentons ici (fig. 't) la chaleur spécifique vraie d'un métal non

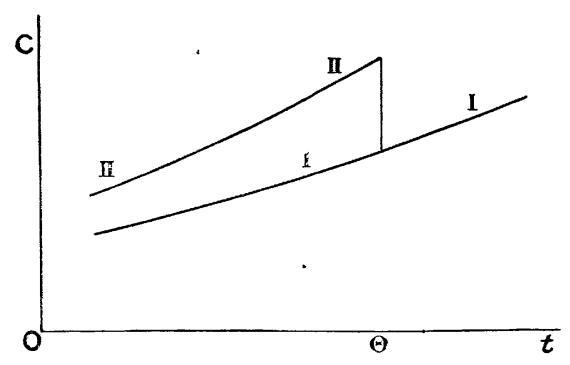

Fig. 4. magnétique. Elle croìtra régulièrement en fonction de la température (courbe I). Ajoutons-y le terme magnétique qui croìt progressivement du zéro absolu au point de Curie, qui en ce point s'annule brusquement et reste nul au-dessus de ce point (courbe II).

La chaleur de transformation, si l'on tient à conserver ce terme, ou mieux, la chaleur de désaimantalion est dépensée dans lout l'intervalle du zéro absolu au point de Curie. En ce point, la chaleur spécifique vraie présente une disconlinuité qui est l'expression $\mathrm{du}$ fait que la désaimantation étant complète il n'y a plus de chaleur de désaimantation à fournir. L'expérience a confirmé cette conséquence de la théorie. 
Il est très frappant que cette théorie qui. par certains côtés, a si bien fail ses preures, présente des insuflisances incontestables.

J'ai comparé dans la figure 3 la variation de l'aimantation spontanée de la magnétite aver la théorie. Nous aurons encore un accord satisfaisant pour le ferronickel répondant à la formule $\mathrm{Fe}^{2} \mathrm{Ni}$. Mais si nous prenons les mélaux purs, le ler ou le nickel, l'expérience nous donne une courbe qui a sans doule la mème physionomie générale, mais qui, quantitativement, s'écarte notablrment de la théorie.

Ce n’est pas (que ce soit un résultat négligeable, que de reprołuire la phỵsionomie générale d'un phénomène jusqu'alors totalement inexpliqué, mais des écarts pareils ne sont pas sans causer un certain malaise et lémontrenl que la théorie demande lout au moins des retouches partielles.

Quant-aux phénomènes au-dessus du point de Gurie, la droite qui représente linverse des coefficients d'aimantation-passe, pour les trois métaux, fer, nickel et cobalt, non par le point de Curie lui-mème, mais coupe l'axe des abscisses à une dizaine de degrés au-dessus. C'est comme sil s'était substitué, au point de Curie de la région ferromagnétique, un point de Curie légèrement plus élevé. La droite rejoint d'ailleurs le point de Curie de la région lerromagnétique par un raccord curviligne. Des contradictions partielles à còté de bonnes vérifications sont, on le sait par expérience, souvent l'origine de nouveaux progrès et' c'est précisémenì le désir de trouver la clef de ces énigmes qui m’a fait attribuer une telle importance au relevé exact des propriétés du nickel.

Mon collaborateur, M. Auguste Piccard, avait imaginé une méthode de mesures nouvelle et avail réalisé l'appareil avec une rare virtuosité. Je vous demande la permission de dire deux mots de la méthode et de l'appareil.

Nous disposions d'un très gros électro-aimant bobiné avec du tube de cuivre. Le courant circulant dans la paroi du tube et l'eau de réfrigéralion dans la cavité cylindrique, la constance de la température était parfaite mème aux plus fortes excilalions.

La figure 5 donne une représentation schématique de l'appareil. P, P sont les pòles de l'électroaimant. Ils sont percés, suivant l'axe, d'un trou cylindrique dans lequel on peut faire glisser parallèlement à l'axe le four électrique, cylindrique lui aussi. qui contient la substance, une sphère s de nickel pur. Autour de ce four se trourent deux bobines bl' fixes, bobinées en sens contraires. On peut, par le mouvement du four. faire passer la sphère du centre de l'une des bobines (position s) au centre de l'autre (position $s^{\prime}$ ). 
Pourquoi cette disposition? Le mouvement qui produit un accroissement de flux dans l'une des bobines et une diminution dans l'autre donnera, par suite du bobinage inverse, des forces électromotrices instanlanées

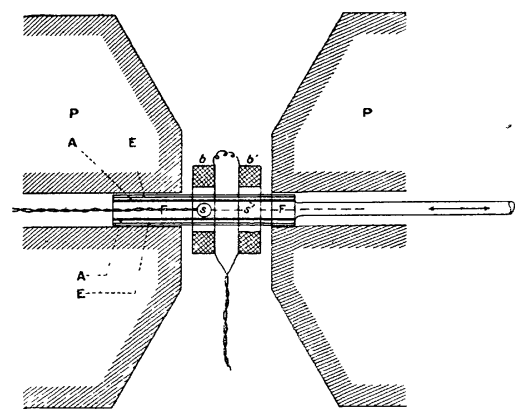

Fig. 5. qui s'ajouteront. Nous pourrons donc, par la méthode balistique, mesurer le moment de cette sphère et, pạr conséquent, son intensité d'aimantation. Grâce à l'artifice des deux bobines enroulées en sens inverses, nous éliminons, ce qui est très important, les effets des petites variations accidentelles d'aimantation de ce gros électroaimant. L'expérience serait à peine praticable sans cette compensation, les quanlités à mesurer étant

incomparablement plus petites que le flux que l'électroaimant envoie à travers l'une des bobines.

Le four électrique $\mathrm{FF}$, dans lequel se trouvait cette petite sphère $\mathrm{s}$, mérite aussi d'ètre décrit. Nous avons trouvé dans l'écume de mer une substance rigoureusement non magnétique et supportant les hautes températures. La carcasse du four, un tube mince en écume de mer, renforcé aux bouts, porte, dans un double filetage de sens contraires, un bobinage bifilaire de tresses de platine pour le courant de chauffe. Il y avait ensuite, autour de ce tube, une gaine d'air A de deux ou trois dixièmes de millimètre, un premier tube de verre mince, d'environ trois dixièmes de millimètre d'épaisseur, une gaine d'eau E, d'environ un demi-millimètre, circulant entre ce premier tube et un second, de façon à entourer le four électrique, protégé par la gaine d'air, d’une enveloppe à température rigoureusement constante,

L'eau alimentant la gaine était privée d'air par ébullition, parce que la moindre bulle de gaz qui șe serait dégagée aurait changé les conditions de refroidissement et empèché l'extrème constance de température qui était indispensable. Cette eau, après avoir été portée à l'ébullition, était conservée dans un réservoir de 100 litres placé dans la mème pièce que l'électroaimant. Le four, avec toutes ses enveloppes, n'avait qu'un diamètre de 1 ŏ millimètres.

Cet appareil recevait, par l'axe de l'électroaimant, le couple thermoélectrique avec ses deux conducteurs, l'amenée du courant, le tube d'amenée de l'eau, le tube de sortie de l'eau, et il y avait encore un tube à succion pour enlever l'eau hygroscopique qui se dégageait de l'écume de mer aụ 
commencemenit de la chaufle. On pouvail maintenir la température constante, pendant plusieurs minutes, à moins d'un cinquantième de degré près, au voisinage de 400 degrés.

La figure 6 représente les isothermes magnétiques obtenues arec cet appareil. Ce sont des courbes d'aimantation au sens usuel du mot, relevées pour une série de températures voisines, dans la région du point de Curie. Cerlaines d'entre elbes, correspondant aux températures relativement basses, sont forlement courbées. Puis, on les voit prendre progressivement la forme rectiligne correspondant à la proportionnalité de l'aimantation au champ. La première courbe, au haut de la figure, cor-

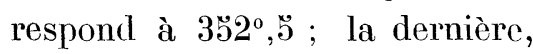
au bas de la figure, à $407^{\circ}, 5$. Le point de Curie caractérisé par la perte du ferromagnétisme spon-

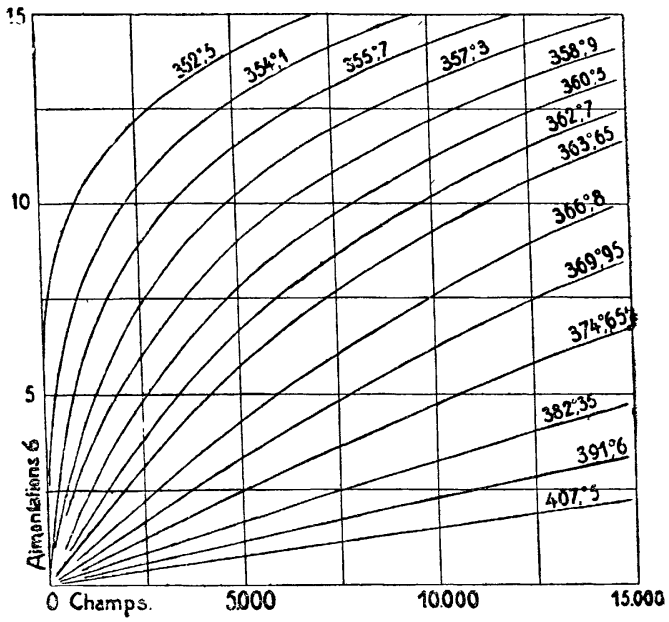

Fig. 6 . tané est à $356^{\circ}, \breve{5}$.

Pour donner une idée de la précision nécessaire dans la mesure des températures, signalons le fait que la différence de température entre deux courbes successives est, dans la plupart des cas, voisine d'un degré et demi et que, si nous voulions déterminer les différences d'aimantation d'une courbe à l'autre avec une précision élevée, par exemple, à 2 pour 100 près, il faudrait aussi que cette différence d'un degré et demi fùt réalisée avec la mème précision, ou mème une précision supérieure, puisqu'elle n'est qu'une des sources d'erreurs possibles.

Dans le développement qui suit, je vais ètre amené à indiquer des propriétés que les expériences déjà faites laissent apparaître. mais qui n'ont pas encore la précision requise pour une publication détaillée. Il est essentiel que j'en fasse connaìtre les grandes lignes pour que nous puissions ensuite discuter le phénomène que je me propose de vous présenter.

La figure 7 donne une représentation différente des mèmes expériences. Ce sont les lignes d'aimantation constante, déduites des isothermes de la figure 6 . Chaque ligne correspond à une valeur donnée de $\sigma$. Les températures ont été portées en abscisses et les ordonnées sont les champs nécessaires pour obtenir cette aimantation $\sigma$. 
La relation entre la température et le champ est linéaire, c'est une grande simplification. Cette propriété est nettement visible dans la figure 7 . Mais, en mème temps, se présente un fait très curieux. Pour une mème aimantalion. cette relation linéaire n'est pas la mème dans toute l'élendue de la figure. Plusieurs de ces lignes sont, en effet, composées de deur

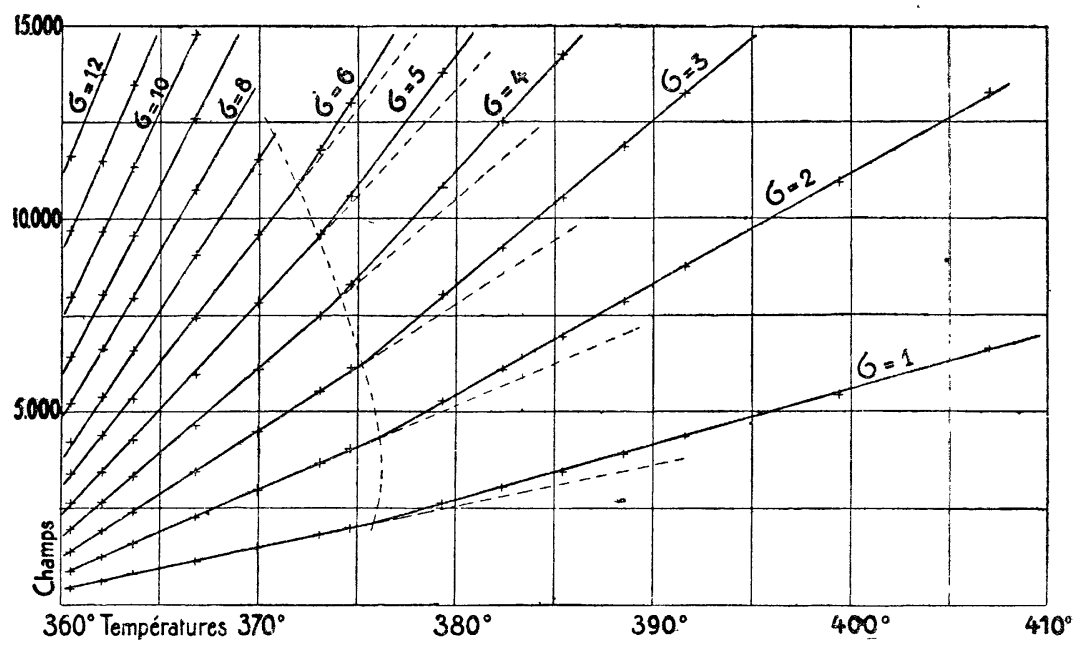

Fig. \%.

segments formant des droites coudées. Le lieu des coudes partage le plan en deux régions dont chacune paraìt c̀tre le domaine d'existence d'un état particulier du nickel. La nature de ces élats est d'ailleurs très énigmatique. On peut dire, cependanl, qu'ils sont caractérisés par des moments atomiques et des constantes du champ moléculaire différents.

On peut tenter d'expliquer. par des changements d'état de cette espèce, l'insuffisant accord de l'expérience et de la théorie. Si le phénomène linéaire aux hautes températures ne commence pas au point de Curie, mais plus loin, cela pourrait ètre parce qu'il y a un ou plusieurs changements d'état au voisinage du commencement de la droite. De mème si des changements d'état, au lieu de se produire comme ici dans le voisinage du point de Curie, ont lieu aux lempératures plus basses, dans la région ferromagnétique, on pourra leur altribuer la divergence entre la courbe théorique de la variation thermique de l'aimantation spontanée et les courbes expérimentales très différentes du fer et du nickel. Des mesures plus précises et plus étendues permettront sans doute de reconnaitre si cette interprétation est exacte.

Nous en étions à l'observation de ces isothermes, lorsqu'il s'est pré- 
senté un phénomène imprévu qui arait l'apparence de quelqu'erreur expérimentale d'origine inconnue. Chaque fois qu'on fermait le courant de l'aimant ou qu'on le coupait, il se produisait un mouvement de l'index lumineux du galvanomètre des températures. Ce mouvement paraissait assez brusque. Mais la technique employée empèchait de se rendre un compte exact de son degré de rapidité et de voir par là à quél effet il était lié. Dans cette expérience, il y a, en effet. toules sortes de causes de ralentissement. Le courant s'établit avec une certaine lenteur par suite de la forte self-induction: l'aimantation de l'électro-aimant est en retard sur le courant d'excitation. Les instruments de mesure ont leur lenteur propre, les varialions de température du four suivent avec un certain retard celles du courant de chauffe. Et la durée de tous ces phénomènes est de l'ordre de grandeur de celle de l'effet observé. Le sens de la déviation observée correspondait à un échauffement à la fermeture et un refroidissement à la rupture du courant d'excitation. Ce phénomène était notable : il se traduisait par un écart de 2 centimètres et mème plus sur l'échelle des températures et revenait à une variation de température allant jusqu'à 7 dixièmes de degré.

Pendant trois semaines, nous avons cherché sans succès à nous expliquer ce phénomène, partagés entre des sentiments divers. Nous songions ou bien à abandonner celle méthode dans laquelle se cachait une cause d'erreur irritante qui, dépassant les erreurs acceptables, menaçait de tout compromettre, ou bien à continuer à tout hasard avec le vague espoir que les choses s'expliqueraient un jour. Nous en étions là lorsqu'une particularité nous mit sur la voie et nous avons continué.

Passant à des isothermes de température notablement plus élevées nous avons vu diminuer à nouveau le phénomène qui, d'abord, avait augmenté avec la température. Il prenait des valeurs de plus en plus faibles à mesure qu'on s'écartait du point de Curie.

Le phénomène inconnu devait donc résider dans le nickel et ètre en rapport avec ses propriétés magnétiques. Cette constatation changea le cours de nos idées; jusqu'alors. en effel, nous avions mis en cause les appareils. D’abord, nous avions soupçonné une action du champ magnétique sur le couple thermoélectrique; à tort, car nous avons pu constater, en effet, que pour les températures et les champs de l'expérience sa force électromotrice est indépendante du champ à 1/70000 au moins de sa valeur.

Nous avions soupçonné une action directe que l'électroaimant exercerait sur le galvanomètre du couple thermoélectrique, une induction sur 
le circuit de ce couple, de la chaleur développée par des courants de Foucault. Nous avions tout contrôlé : aucune de ces hypothèses ne s'était vériliée.

Une fois sur la voie, j'ai eu l'impression que le phénomène devait être une autre manifestation de la mème donnée énergétique qui fait que les chaleurs spécifiques des ferromagnétiques ont leur physionomie propre. Et, en effet, par un calcul faisant intervenir le champ moléculaire, j'ai trouvé qu'il devait effectivement y avoir échauffement par l'aimantation, refroidissement par la désaimantation et que l'ordre de grandeur était celui que l'expérience avait donné. Dorénavant il ne s'agissail plus de rechercher une cause d'erreur, mais d'étudier un phénomène nouveau.

Nous pouvons nous rendre compte, maintenant, qu'en effet il était facile à prévoir. Considérons la formule thermodynamique de la chaleur élémenlaire à donner à une substance qu'on aimante. Cette chaleur $\mathrm{d} Q$ se compose, d'abord, d'un terme indépendant de toute variation de l'état magnétique, qui s'exprime par la chaleur spécifique à aimantation constante $C_{\sigma}$, dont la chaleur spécifique ordinaire des substances non magnétiques n'est que le cas particulier correspondant à l'aimantation nulle.

On a donc

$$
\mathrm{d} Q=C_{\sigma} \mathrm{d} t
$$

Si la substance s'aimante, il s'y ajoute un terme qui provient de la variation de l'aimantation $\sigma$.

Lorsque, comme dans le cas des gaz paramagnétiques, l'énergie ne dépend pas de l’orientation des molécules les unes par rapport aux autres, tout le travail $H_{e} \mathrm{~d} \sigma$ fourni par le champ extérieur $H_{e}$ se transforme en chaleur qui doit ètre enlevée pour que la température reste constante, donc :

$$
\mathrm{d} Q=C_{\sigma} \mathrm{d} t-H_{e} \mathrm{~d} \sigma .
$$

Même dans les gaz paramagnétiques l'aimantation adiabatique serait accompagnée d'une variation de température. En effet, $\mathrm{d} Q=0$ donne

$$
\mathrm{d} t=\frac{H_{e}}{C_{\sigma}} \mathrm{d} \sigma .
$$

Cet effet serait extrèmement faible.

Lorsqu'il y a un champ moléculaire $H_{m}$ le travail qu'il fournit s'ajoute au travail du champ extérieur et l'on a : 


$$
\mathrm{d} Q=C_{\sigma} \mathrm{d} t-\left(H_{e}+H_{m}\right) \mathrm{d} \sigma\left(^{1}\right) .
$$

Or, l'étude magnétique du nickel montre que, pour toutes les valeurs đe $\sigma$ qui ont été atteintes, le champ moléculaire est donné par

$$
H_{m}=n \sigma,
$$

où $n$ est une constante. Donc,

$$
\mathrm{d} Q=C_{\sigma} \mathrm{d} t-\left(H_{e}+n \sigma\right) \mathrm{d} \sigma .
$$

Cherchons d'abord le terme magnétique de la chaleur spécifique. Quand on chauffe une substance ferromagnétique dans un champ extérieur nul, l'aimantation spontanée décroît (fig. 3), $\sigma$ est une fonction de $t$ prescrite par cette décroissance et la chaleur spécifique donnée par l'expérience est

$$
C=\frac{\mathrm{d} Q}{\mathrm{~d} t}=C_{\sigma}-n \sigma \frac{\mathrm{d} \sigma}{\mathrm{d} t}
$$

ou

$$
C=C_{\sigma}-\frac{n}{2} \frac{\mathrm{d}\left(\sigma^{2}\right)}{\mathrm{d} t}
$$

Le terme qui, pour les ferromagnétiques, s'ajoute à la chaleur spécifique ordinaire est donc

$$
C_{m}=-\frac{n}{2} \frac{\mathrm{d}\left(\sigma^{2}\right)}{\mathrm{d} t}
$$

qui est positif puisque $\tau^{2}$ décroît quand la température s'élève.

$\mathrm{Au}$ lieu de mesurer des chaleurs spécifiques recherchons maintenant les variations de température dans une opération rapide, durant laquelle les échanges de chaleur avec le milieu ambiant ne peuvent se produire. Il faut donc, dans l'équation (8) faire $\mathrm{d} Q=0$.

(1) Si l'on remplace l'hypothèse primitivê du champ moléculaire par l'hypothèse plus gẻnéra le (Voir page 163, note 1.)

$$
H_{n}=-\frac{\partial U}{\partial \sigma},
$$

où $U$ est l'énergie de la substance, on retrouve la même équation. En effet, en appelant d $\mathfrak{G}$ le travail fourni par le champ extérieur, on a :

mais

$$
\mathrm{d} Q+\mathrm{d} \mathscr{G}=\mathrm{d} U=\frac{\partial U}{\partial \sigma} \mathrm{d} \sigma+\frac{\partial U}{\partial t} \mathrm{~d} t
$$

d'où l'équation (8).

$$
\frac{\partial U}{\partial \sigma}==-H_{m}, \quad \frac{\partial U}{\partial t}=C_{\sigma} \quad \text { et } \quad \mathrm{d} \mathscr{G}=H_{\boldsymbol{e}} \mathrm{d} \sigma
$$


Pour achever le calcul, remarquons que, dans les limites de nos expériences. qui vont jusqu à $\sigma=16$, l'aimantation est proportionnelle au champ total $\boldsymbol{H}+\boldsymbol{H}_{\boldsymbol{m}}$. Le quotient est le coefficient d'aimantation, c'est-à-dire la constante de Curie $C$ divisée par la température absolue, donc

$$
\frac{\sigma}{H+H_{m}}=\frac{C}{T}
$$

et, en tenant compte de la relation,

$$
\text { C.n }=\Theta
$$

qui exprime que l'aimantation spontanée disparait au point de Curie, l'équation (8) donne:

$$
\mathrm{d} t=\frac{T}{\Theta} \frac{n}{2 C_{\sigma}} \mathrm{d}\left(\tau^{2}\right)
$$

Il se produit donc une élévation de température sensiblement proportionnelle à l'accroissement du carré de l'aimantation. Le facteur de proportionnalité comprend en effet un terme $\frac{\cdot n}{2 C_{\sigma}}$ constant, dans la mesure où la . chaleur spécifique $C_{\sigma}$ est constante, et le facteur $\frac{T}{\Theta}$ qui varie peu dans la région peu étendue autour du point de Curie, où, comme nous le verrons, le phénomène existe avec une certaine ampleur.

C'est bien le phénomène que nous avons observé, quant-à la nature de l'effet et au signe : élévation de température lorsque l'aimantation s'accroìt, abaissement lorsqu'elle diminue par l'établissement et la suppression du champ. Nous avions tout ce qu'il fallait pour deviner l'existence de ce phénomène magnétocalorique.

Ce phénomène a une parenté facile à établir avec d'autres, dont on s'occupe en thermodynamique. Vous sarez que, lorsqu'on exerce une traction sur une barre de fer, cette barre de fer se refroidit. Lorsqu'on comprime la même barre de fer, elle s'échauffe. La traction, l'allongement de la barre de fer sont liés à une diminution de densité. Lorsque, par un effet mécanique, la densité du fer diminue, la barre se refroidit. Cela tient à ce que, lorsque les molécules s'écartent, l'accroissement correspondant d'énergie inlerne n'est fourni qu'en partie par le travail extérieur et que le déficit est comblé par le réservoir thermique. Inversement, lorsqu'on augmente la densité, il y a de l'énergie potentielle versée dans le réservoir thermique et la matière s'échauffe.

L'aimantation n'est-elle pas aussi une rariation de densité ? Oui, mais ce n'est pas la densité en volume, c'est la densilé en direction. 
Considérons les molécules avec leurs moments magnétiques, et traçons les rayons d'une sphère, parallèles à la direction du moment de chacune d'entre elles. Nous obtiendrons ainsi une image de leur distribution en direction. Lorsque les moments seront dirigés dans tous les sens, toute la sphère sera couverte uniformément par les points figuratifs. Leur densité sera faible. Aimantons la substance : les directions se ramassent, les molécules orientées forment un faisceau plus ou moins étroit, les points figuratifs se rassemblent en certaines régions de la sphère la densité augmente. Le phénomène observé peut donc s'énoncer : lorsque la densité en direction s'accroît, la substance s'échauffe; lorsqu'elle diminue, la substance se refroidit. Il y a parallélisme complet avec le phénomène mécanique.

Passons maintenànt à l’examen plus détaillé des résultats de l'expérience, figure 8 . On a porté en ordonnées la grandeur du phénomène magnétocalorique, expérimental, exprimée par les variations de tem-

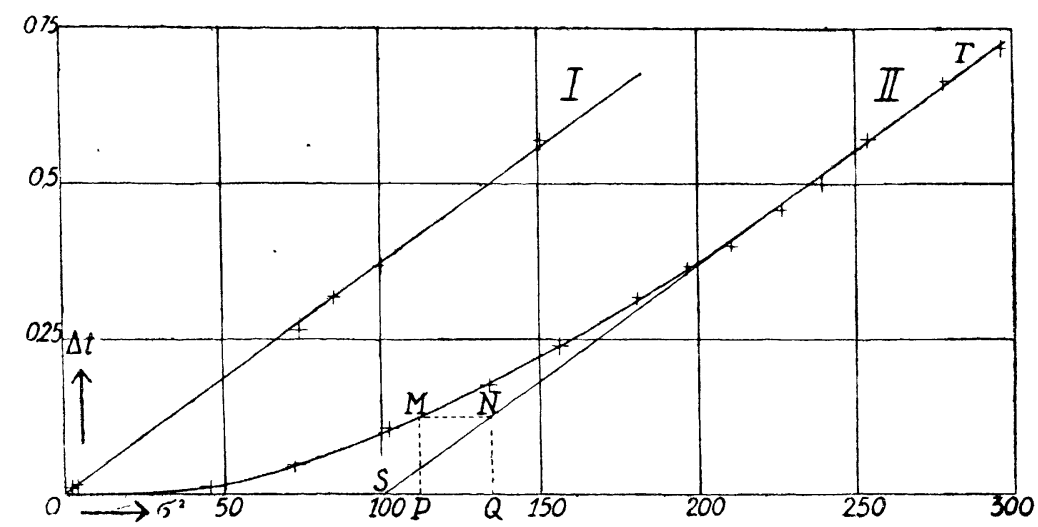

Fig. 8.

pérature de la substance, en degrés centésimaux. Toute la hauteur de la figure correspond à $3 / 4$ de degré. Les carrés des aimantations $\sigma^{2}$ ont été portés en abscisses. Portons d'abord notre attention sur la courbe I. On voit que l'ensemble des points observés, marqués par des croix, définit assez bien une droite passant par lorigine. Ces observations sont reproduites dans le tableau I.

Dans nos expériences, à la fermeture ou à la rupture du courant de l'aimant, $\sigma^{2}$ varie de zéro à la valeur indiquée et réciproquement. La proportionnalité donnée par la droite et par la dernière colonne du tableau I vérifie notre théorie.

Ces observations se rapportent à la température de $361^{\circ}, 8$ de l'échelle 
Tableid I

\begin{tabular}{|c|c|c|c|}
\hline$H$ & $\Delta t^{0}$ & $\sigma^{2}$ & $\frac{\Delta t}{\sigma^{2}}$ \\
\hline 990 & 0.011 & 2.18 & $(0,00.0 \ddot{3})$ \\
\hline 1320 & 0,014 & 4,01 & $(0,00347)$ \\
\hline 7820 & 0,264 & דタ, & $0,0035 \%$ \\
\hline 8780 & 0,317 & 800,50 & 0,00371 \\
\hline $100 \% 0$ & 0,370 & 100,8 & 0,00367 \\
\hline 14960 & 0,369 & 1501,0 & 0,00377 \\
\hline
\end{tabular}

ordinaire, nettement plus élevée que le point de Curie observé pour la mème substance à $356^{\circ}, \breve{s}$. Aux températures supérieures au point de Curie, il n'y a pas d'aimantation spontanée. Toute aimantation prend effectivement naissance au moment de l'application du champ. Il n'y a pas d'ambiguïté.

Par contre, au-dessous du point de Curie où l'aimantation spontanée existe, l'effet du champ est double. Il y a un changement d'orientation de l'aimantation préexistante, qui ne doit contribuer en rien au phénomène, et, si le champ est suffisant, un changement de sa grandeur, qui seul est efficace. Et effectivement, la courbe II qui se rapporte à la température de $352^{\circ}, 1$ exprime bien cet état de choses. Au commencement, tant que nous ne faisons qu'orienter l'aimantation existante, le carré de l'aimantation donnée par l'observation augmente, mais l'élévation de température ne se produit pas ; ensuite elle commence à apparaitre, faiblement d'abord parce qu'une faible augmentation de la vraie grandeur commence à accompagner l'orientation. Puis cet effet s'accentue et la courbe se relève de plus en plus. Quand, pratiquement, l'aimantation spontanée a pris la direction du champ, tous les accroissements observés de $\sigma^{2}$ sont vrais et sont accompagnés d'une élévation de température qui leur est proportionnelle avec le même facteur de proportionnalité qu'au dessus du point de Curie. La courbe II tend à devenir parallèle à la droite I. Le phénomène s'adapte donc remarquablement aux notions acquises, par ailleurs, d'aimantation spontanée, d'orientation subséquente et d'accroissement ultérieur par un champ de plus en plus fort. Les observations avec lesquelles a été construite la courbe II sont données dans le tableau II.

Nous allons pouvoir nous servir du phénomène magnéto-calorique pour déterminer la vraie grandeur de l'aimantation. On peut inteŕpréter les courbes I et II de la figure 8 en disant que les abscisses représentent les valeurs apparentes du carré de l'aimantation et les ordonnées ses valeurs 
Tibleiv II

\begin{tabular}{|c|c|c|}
\hline II & $\Delta t^{\prime}$ & $\sigma^{3}$ \\
\hline 320 & 0.011 & 1.3.8 \\
\hline $\mathbf{6 6 0}$ & 0,048 & 72,8 \\
\hline 1320 & 0,106 & 102,3 \\
\hline 2330 & 0,176 & 131, З \\
\hline 3340 & 0,290 & $1 \check{6} 6,2$ \\
\hline 4670 & 0,319 & 181,2 \\
\hline$\ddot{3010}$ & 0,367 & 197 \\
\hline 6000 & 0,399 & 211 \\
\hline 7820 & 0 , גי8 & 227 \\
\hline 8780 & $0, .500$ & $239, \tilde{3}$ \\
\hline $100 \% 0$ & $0, \square 72$ & $2 \ddot{4}$ \\
\hline 12320 & $0,66.5$ & 278 \\
\hline 11230 & 0,718 & נ艹 \\
\hline
\end{tabular}

vraies à une autre échelle. Quand il y a une aimantation spontanée, ces dernières sont comptées à partir du carré de cette quantité. Si l'aimantation était constamment orientée dans la direction du champ, la courbe II serait remplacée par la ligne OST, où OS est le carré de l'aimantation spontanée. Comme ST peut ètre tracé au moyen des données expérimentales, il en résulte que la mesure du phénomène magnétocalorique, combinée avec les mesures d'aimantation, donne sans ambiguïté l'aimantalion spontanée.

Je puis, sans entrer dans les détails, ajouter que les trois déterminations que j'ai faites pour trois températures différentes ont donné une concordance salisfai:ante avec les déterminations par les méthodes purement magnétiques, plus indirectes.

Mais ce n'est pas tout. Soit OP une valeur observée du carré de l'aimantalion apparente; PM représentera l'excès du carré de l'aimantation vraie sur le carré de l'aimantation spontanée, à l'ćchelle des ordonnées. Reportons cet excès en $\mathrm{N}$ sur la droite ST. L'abscisse $O Q$ donnera le carré de l'aimantation vraie correspondant à cet excès, c'est-à-dire à la valeur $\mathrm{OP}$ du carré de l'aimantation apparente dont nous sommes partis. On pourra donc, au moyen du phénomène magnétocalorique, par une réduction facile, tracer, à còté du réseau des isothermes de l'aimantation apparente, celui des isothermes de l'aimantation vraie.

Examinons maintenant la figure 9 dans laquelle j’ai réuni, dans la mème représentation que figure 8 , tous les résultats obtenus jusqu'à présent avec l'appareil décrit. Nous retrouvons en A la droite I de la figure 8. 
Viennent ensuite les trois courbes B, C, D correspondant à trois températures échelonnées à 1,6 degré l'une de l'autre.

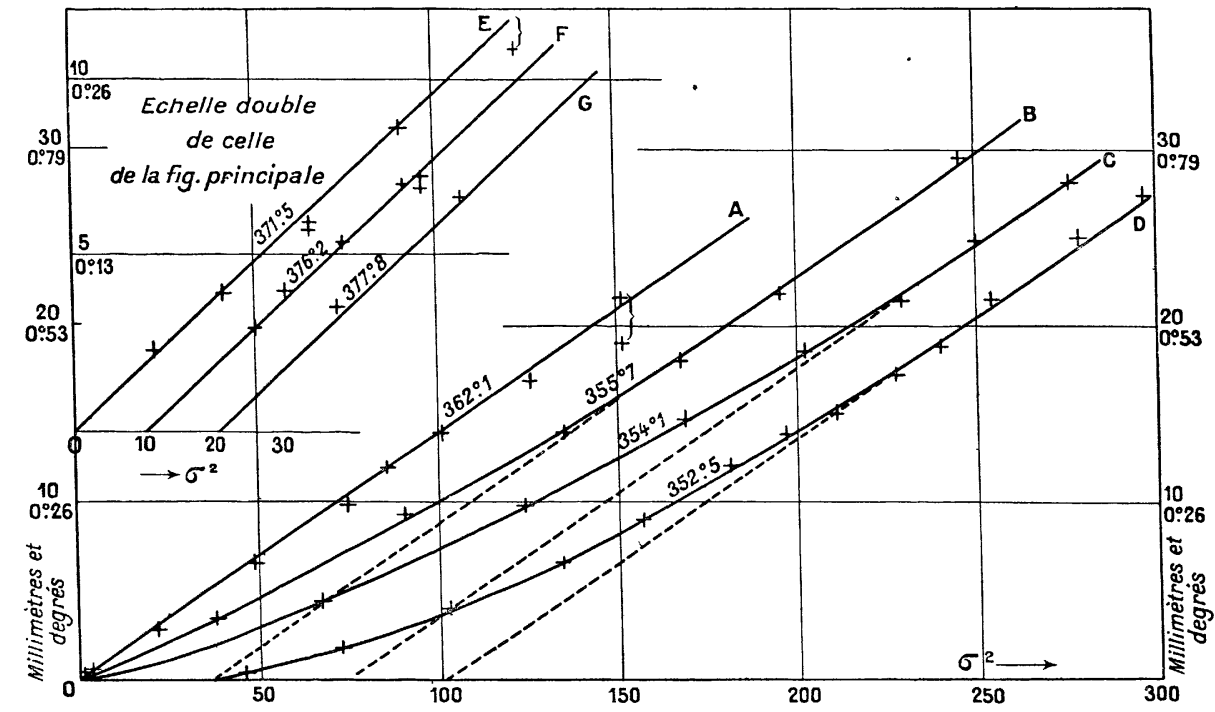

Fig. 9 .

On voit que la partie de la courbe qui correspond aux grandes valeurs de $\sigma^{2}$ tend toujours à devenir parallèle à la droite $\mathrm{A}$.

Ces quatre courbes correspondent à quatre lempératures qui sont situées dans la partie gauche de la figure 7 où les lignes d'égale aimantation sont représentées par des lignes brisées dont les coudes sont sur la ligne de séparation de deux états de la substance. En haut et à gauche de la figure 9 , ont été réunis des résultats sur le phénomène magnétocalorique obtenus à des températures plus élevées, de l'autre côté de la ligne de séparation. Les aimantations atteintes étant plus petites, le phénomène est plus faible, moins facile à observer, mais il a encore le même aspect. La droite $\mathrm{F}$ correspondant à $376.2^{\circ}$ a été considérée comme la mieux déterminée. C'est elle qui a donné le rapport de l'élévation de température à $\sigma^{2}$. Les deux autres droites $\mathrm{E}$ et $\mathrm{G}$ correspondant à $372^{\circ}, \mathrm{g}^{\circ}$ et $377^{\circ}, 8$ ont été menées parallèlement à la première. Ce tracé s'accorde dans les limites des erreurs possibles avec les données expérimentales.

En fait, nous avons étudié le phénomène magnétocalorique dans une région restreinte, mais sur deux substances qui, toutes deux, sont des états du nickel. Pour chacune, nous possédons l'inclinaison de la droite qui représente la relation entre l'élévalion de température et $\sigma^{2}$. 
Le calcul de l'élévation de température peut se faire à priori d'après la formule (11), simplifiée en remarquant que pour toutes nos mesures la température $T$ est roisine du point de Curie $\Theta$ :

$$
\mathrm{d} t=\frac{n}{2 C_{\sigma}} \mathrm{d} \sigma^{2},
$$

qui suppose la connaissance du cocflicient $n$ du champ moléculaire et de la chaleur spécifique à aimantation constante. Pour la partie gauche de la figure 7 les mesures magnétiques donnent $n=67600$; pour la partie droite, 90700 . Quant-à la chaleur spécifique $C^{\sigma}$, elle est donnée par la chaleur spécifique vraie, mesurée immédiatement au-dessus du point de Curie. J'emprunte sa valeur, $C=0,1256$ en calories et $C=0,1256 \times{ }^{4}, 19 \times 10^{7}$ en ergs à un travail de Weiss, Piccard et Carrard $\left(^{1}\right)$. On trouve, pour le premier état du nickel,

$$
\mathrm{d} t=0,642 \cdot 10^{-2} \mathrm{~d} \sigma^{2}
$$

et, pour le second,

$$
\mathrm{d} t=0,862 \cdot 10^{-2} \mathrm{~d} \sigma^{2} .
$$

Mais l'expérience doit nous donner des valeurs plus petites, pour deux raisons. D'abord, à cause de la lenteur de l'établissement du chañp, le phénomène ne pourait ètre instantané. Il fallail attendre qu'il fùt achevé pour faire l'observation. Nous avons estimé cu'il l'était. pratiquement, après 25 secondes. Pendant ce lemps une partie de la chaleur dégagée se perd. Mais si l'on prend soin de donnor à cette période d'attente exactement la mème durée, la chaleur perdue sera la mème fraction de la chaleur dégagée.

Ensuite, le contact du couple arec la sphère de nickel était établi au moyen d'une petite calotte d'argent qui augmentait la capacité calorifique. On peut évaluer grossièrement à 13 pour 100 la perte de l'élévation de température qui en résultait.

Pour le premier état du nickel l'expérience donne

$$
\mathrm{d} t=0,368.10^{-2} \mathrm{~d} \sigma^{2},
$$

au lieu de

$$
\mathrm{d} t=0,642 \cdot 10^{-2} \mathrm{~d} \sigma^{2}
$$

et, pour le second,

au lieu de

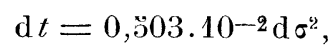

$$
\mathrm{d} t=0,862 \cdot 10^{-2} \mathrm{~d} \sigma^{2} .
$$

(1) Arch. des Sc. phys. et nat., 1917, t. xuII, p. 11 \%. 
Dans le premier cas la valeur observée est 0,57 1 : dans le second, 0,583 de la valeur,calculée. Les valeurs observées sont plus faibles comme il convient. Mais la concordance des nombres ci-dessus montre que le rapport des nombres observés pour les deux élals est, avec une précision plus grande qu'on ne pouvait l'attendre, idenlique au rapport des nombres calculés. Il y a là un commencement de vérification très satisfaisant.

La figure 10 donne, sous une autre forme, les mèmes résultats expéri-

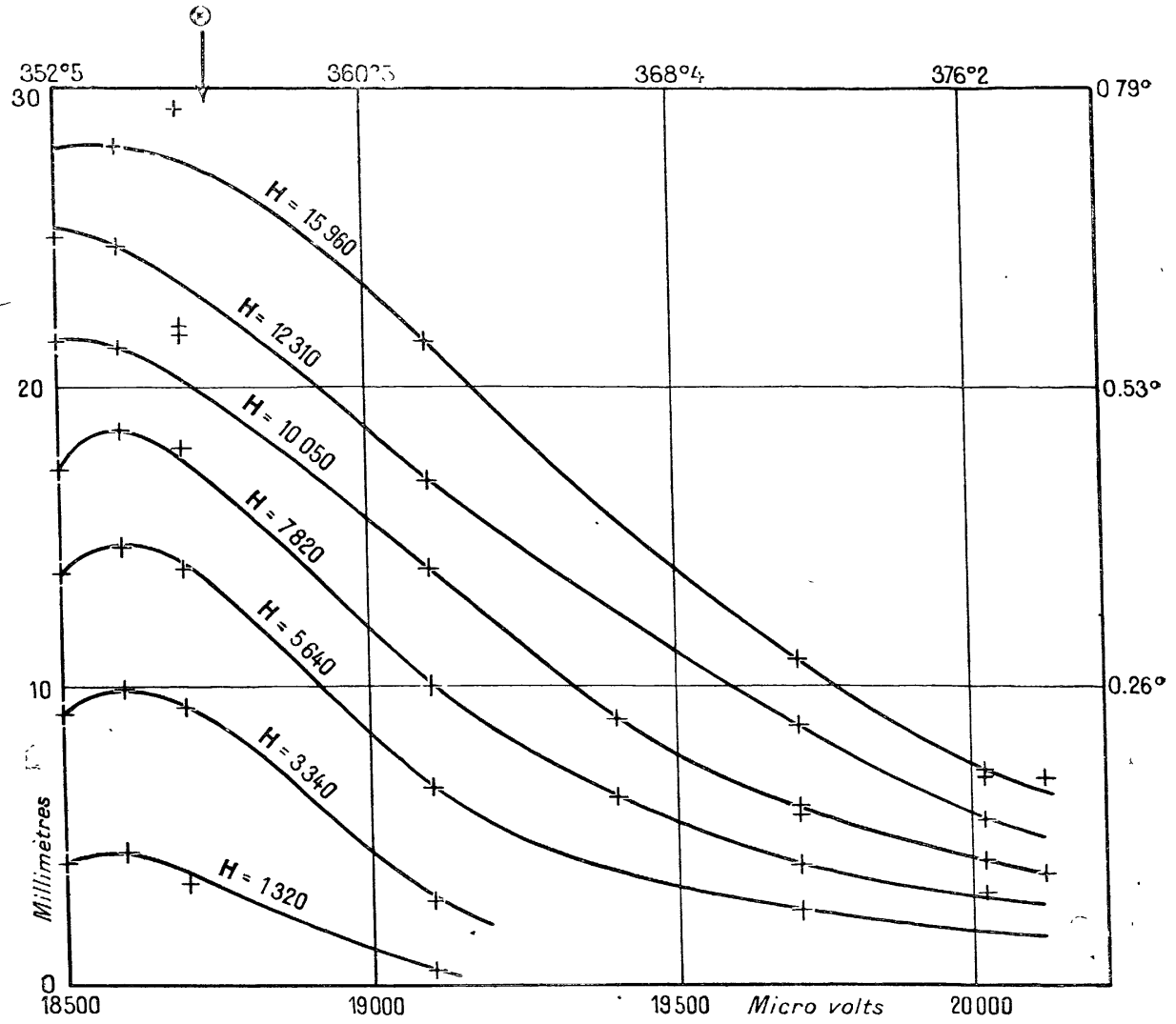

Fig. 10 .

mentaux que la figure 9. Chacune des courbes se rapporte à une excitation déterminée de l'électro-aimant.

Les températures, représentées par les forces électromotrices du couple, ont élé portées en abscisses; les phénomènes magnétocaloriques, en millimètres de l'échelle, en ordonnées. En haut et à droite de la figure ont été inscrites quelques valeurs des abscisses el ordonnées en degrés centésimaux. Le point de Curie est marqué par une flèche et la lettre $\Theta$. 
Gomme nous le savions, le phénomène a une ampleur particulière dans la région du point de Gurie et, quand la température s'élève à partir de ce point, il décroil très rapidement. C'est la particularité qui nous arait mis sur la voie de sa découverte.

Dans cetle série de mesures, la température la plus basse est encore assez voisine du maximum. Relativement à ce qui se passe au-dessous du point de Curie, on ne possède que des renseignements assez sommaires : c'est une série de mesures rapides embrassant tout l'intervalle de $23^{\circ}$ à $500^{\circ}$ qui a été faite avec un autre four électrique sur un autre échantillon de nickel et une seule valeur du champ $\boldsymbol{H}=22950$ gauss. Le couple constantan-argent n'avait pas été étalonné. Les températures ont été déterminées approximativement en reportant sur ce couple l'étalonnage d'un autre couple formé des mèmes mélaux. Quelque sommaires qu'elles soient, ces expériences, représentées dans la figure 11, donnent une idée de l'allure du phénomène, notamment dans la région jusqu’ici inexplorée, au dessous du

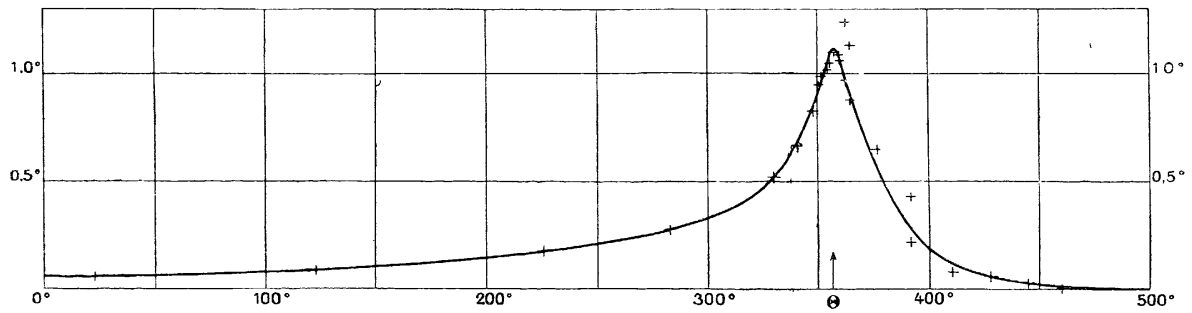

Fig. 11 .

point de Curie jusqu'à la tempéralure ordinaire. Dans ce champ plus intense, le phénomène a dépassé $1^{\circ}$.

La démonstration expérimentale du phénomène magnétocalorique serait facile s'il ne fallait, pour l'observer avec une certaine ampleur, porter la substance à une température voisine du point de Curie, maintenir cette température constante et mesurer exactement ses variations. Elle serail beancoup plus simple pour une substance dont le point de Curie serail à la température ordinaire. Tel est le cas de certains ferronickels irréversibles. de titre en nickel roisin de 25 ou 30 pour 100 , quand par une élévation de température préalable on les a amenés à l’état non magnétique à la température ordinaire. Nous avons examiné, à cet effet, une pelite collection de ferronickels que nous devons à M. Ch.-Ed. Guillaume et nous avons trouvé qu'un métal contenanl 27 pow 100 de nickel donne, dans un champ de 21000 gauss, un elfet de $0^{\circ}$, $34^{\prime}$ environ qui varie peu dans l'intervalle de $8^{\circ}$ à $37^{\circ}$. Avec 
cette substance, la démonstration du phénomène magnétocalorique devient une expérience de cours des plus faciles.

Un couple thermoélectrique argent-constantan est inséré dans un petil cylindre de la substance pesant 1 gramme environ. Ge cylindre est fixé à l'extrémité d'un levier qui permet de l'introduire dans le champ magnétique et de l'en extraire. Il est protégé par une enveloppc de colon contre les variations accidentelles de température. Le couple est relié à un galvanomètre à cadre mobile de faible résistance et à indications relativement rapides. On observe facilement l'échauffement et le refroidissement accompagnant l'introduction dans le champ el l'extraction.

Il reste beaucoup à faire pour achever l'étude du phénomène magnélocalorique. Mème pour le nickel, le travail est à peine ébauché et, bien que la région roisine du point de Curie, particulièrement intéressante, soit ici facilement accessible, il y aura à surmonter de réelles difficultés. Il faut, en effet, que la température soit parfaitement constante au moment où le phénomène se produit; il faul tenir compte des inconvénienls et des incertitudes qui résultent de la lenteur de l'établissement des champs intenses. Il est en outre nécessaire, pour pouvoir pousser la discussion à fond, de mesurer en mème temps, sur le mème échantillon, les valeurs de $\sigma$.

Par le fait mème que je ne parle que du nickel (et d'un ferronickel), vous devinez qu'on ne possède encore aucune donnée sur les autres ferromagnétiques. Il faudra aussi, malgré les difficultés, faire cette étude sur le fer, sur le cobalt, sur d'autres substances, non seulement pour son intérèt propre mais aussi à cause des questions connexes du champ moléculaire et de la vraie grandeur de l'aimantation.

Je me propose, vous le pensez bien, de poursuivre ces recherches et je serais heureux de ne pas ètre seul à les continuer. Mais ce serait pour moi une satisfaction particulièrement vive de voir l'un de vous, Messieurs, un étudiant de l'Université de Bruxelles, venir à Strasbourg prendre sa parł de cette ouvre. 